COMMENT. The diagnostic criteria for pseudotumor cerebri, also termed "Idiopathic Intracranial Hypertension" (IIH), include the following: headache, papilledema, and other symptoms/signs of increased intracranial pressure (ICP), CSF pressures of $>250 \mathrm{~mm} \mathrm{H}_{2} \mathrm{O}$, normal CSF findings, no or only false neurologic localizing signs (eg VIth nerve palsy), normal brain imaging, an awake and alert patient, and no identifiable cause of increased ICP (Dandy criteria reviewed by Binder DK et al. Neurosurgery March 2004;54:538-552). Increased ICP caused by venous sinus thrombosis, sleep apnea, or choroid plexus papilloma is termed a "secondary pseudotumor syndrome." Theories advanced in pathogenesis of IIH include: 1) excess CSF production; 2) increase in cerebral blood volume or brain water content; 3) disturbed CSF absorption secondary to increased sagittal sinus pressure; and 4) endocrinological dysfunction related to obesity and female preponderance. Among secondary causes, the association of increased ICP with dural venous sinus thrombosis is long recognized as "otitic hydrocephalus" secondary to otitis and mastoiditis (Foley J. Brain 1955;78:1-41). Magnetic resonance venography should exclude sinus thrombosis in atypical patients with suspected IIH. Other secondary causes for pseudotumor are those associated with medications and with systemic disease. Medications include vitamin A, antibiotics (eg tetracycline), sulfa drugs, growth hormone, oral contraceptives, corticosteroid withdrawal, and lithium. Systemic diseases associated with IIH include systemic lupus erythematosus, malignancies, Addison's disease, thyroid disease, uremia, and various anemias, including iron deficiency in childhood (Yager JY, Hartfield DS. Pediatr Neurol 2002;27:85-92) and SCD SC in a pregnant adult (Thomas E. Obstet Gynecol 1986;67(3 suppl):75-95). The above report is the first case of PC with SCD-SS. Symptoms of PC abate with the correction of iron-deficiency anemia, and in the above 3 cases of SCD, symptoms resolved regardless of a persistent chronic anemia.

\title{
TRIPTANS DO NOT INCREASE RISK OF STROKE IN MIGRAINE
}

The incidence of stroke, cardiovascular events, and death among 63,575 patients with migraine was compared in those prescribed a triptan $(13,664,21.5 \%)$, non-triptan-treated, and matched nonmigraine control subjects $(77,239)$ identified from the General Practice Research Database by researchers at the Institute of Neurology, University College, London, UK, and Pfizer Inc, New York, NY. The mean observation periods were 35.6 months for migraine patients and 33.3 months for controls. Mean number of triptan prescriptions was 8.6 (range 1-316). Ergotamine treatment was also used in 582 (4.3\%) of triptan-treated and 1,598 $(3.2 \%)$ of non-triptan-treated group. Triptans (at least 3 prescriptions) were not associated with an increased risk of stroke, serious cardiovascular events, or death in this cohort of men and women patients between 15 and $60+$ years. A small increased risk of stroke occurred in migraineurs not treated with triptan. The association between migraine and stroke decreased with increasing age at stroke. An association with migraine and stroke was observed across all age and sex groups, with the strongest associations in men aged 45 to 59 years and women of child-bearing age. Triptans were prescribed to those less at risk of stroke and myocardial infarction. (Hall GC, Brown MM, Mo J, MacRae KD. Triptans in migraine. The risks of stroke, cardiovascular disease, and death in practice. Neurology February (2 of 2) 2004;62:563-568). (Reprints: Dr G Hall, Grimsdyke House, Ravenscroft Park, Barnet, EN5 4ND, UK). 
COMMENT. Migraine is a rare precipitant of acute ischemic stroke, mostly occurring in young women in association with attacks of migraine with aura (see Progress in Pediatric Neurology III, PNB Publishers, 1997;174-5). Triptans constrict coronary arteries, and triptan-induced chest pain may mimic angina. Triptans are contraindicated in patients with ischemic heart disease, and some uncontrolled reports of triptan-associated stroke have limited their use in some cases of migraine. The above controlled study has shown that in general practice, triptan treatment in migraine does not increase the risk of stroke, as long as the treatment is used in patients less at risk of stroke. A slightly higher risk of stroke in the non-triptan-treated group with migraine is noteworthy. The safety and efficacy of triptans has not been established for use in children (PDR 2003).

\section{HEADACHE WITH BENIGN ALTERNATING HEMIPLEGIA}

An 8-year-old boy with benign familial nocturnal alternating hemiplegia of childhood (BNAHC) and severe headache and vomiting during episodes is reported from the University of Rochester, NY. Episodes of nocturnal hemiparesis began at 3 years of age, and they occur several times a week to once a month. They are complicated by dystonic posturing of hands and feet, and they last 20 minutes to 5 hours (average, 2 to 3 hours). Headache that begins after 20 minutes following onset of hemiparesis lasts up to 7 hours, and is followed by fatigue. Triggers include physical and emotional stress and sleep deprivation, while sleep alleviates the symptoms. Medications (flunarizine, phenytoin, valproate, carbamazepine, and ibuprofen) and elimination of migraine dietary triggers were ineffective. EEGs showed contralateral hemispheric slowing during or after an attack. Type 1 diabetes with one generalized hypoglycemic seizure, and hyperactive and oppositional behavior with symptoms of depression and threatened suicide developed later. The family history was positive for migraine (in the mother and father), seizures (great-grandmother), and depression and bipolar disease, but negative for hemiparesis or dystonia. The authors tabulate the clinical characteristics of 6 patients with BNAHC previously reported, 6 having episodes of flaccid hemiparesis, 1 also dystonic and ataxic, 5 having a family history of migraine, and 3 being hyperactive (Andermann E et al. 1994; Chaves-Vischer et al. 2001). (Kavanaugh M, Myers GJ. Benign alternating hemiplegia of childhood: new features and associations. Neurology February (2 of 2) 2004;62:672). (Reprints: Dr Gary J Myers, University of Rochester Medical Center, 601 Elmwood Ave, Box 631, Rochester, NY 14642).

COMMENT. Severe headache and vomiting during episodes were described as unique features in this patient with benign alternating hemiplegia of childhood (BNAHC). The initial report of this syndrome by Andermann E et al. 1994 (see Progress in Pediatric Neurology III, PNB Publ, 1997;171-2) described two brothers with recurrent attacks arising out of sleep, distinguishable from the classic, sporadic form of $\mathrm{AHC}$ having a poor prognosis. Both infants with BNAHC awoke screaming $1 \frac{1}{2} \mathrm{hrs}$ after falling asleep and were paralyzed on one side. After returning to sleep and awakening in the morning, they had recovered. Similar episodes recurred with increasing frequency. Features such as hypotonia, dystonia, and eye movements, characteristic of AHC, were absent. Development remained normal. Both parents suffered from migraine. The etiology of BNAHC is unknown, but it is usually classified as a channelopathy and migraine variant. 